\title{
Operación de los molinos del tándem cañero a dos niveles de presión hidráulica
}

\section{Sugarcane Tandem Mills Operation at Two Hydraulic Pressure Levels}

\author{
Jorge Michel Corrales-Suárez \\ Centro de Estudio de Energía y Procesos Tecnológicos \\ Facultad de Ciencias Técnicas \\ Universidad de las Tunas \\ Correo:jorgecs@ult.edu.cu \\ José Marcos Gil-Ortiz \\ Centro de Estudio de Energía y Procesos Tecnológicos \\ Facultad de Ciencias Técnicas \\ Universidad de las Tunas \\ Correo:jgil@ult.edu.cu
}

Pedro Dionisio Remédios-Castañeiras

Centro de Estudio de Energía y Procesos Tecnológicos

Facultad de Ciencias Técnicas

Universidad de las Tunas

Correo:pdremedio@ult.edu.cu

\author{
Yurisleydis Masjuan-Leyva \\ Dirección de Operaciones \\ Empresa Logística Agropecuaria \\ Correo:yml@ela.lastunas.cu \\ José Alexander Gil-Ceballo \\ Departamento de programación \\ Empresa Nacional de Desarrollo de Software, las Tunas \\ Correo: jgilc@lt.desoft.cu
}

Información del artículo: recibido: octubre de 2013, aceptado: enero de 2014

\section{Resumen}

Entre las áreas de mayor consumo de energía en una central azucarera se encuentra el tándem de molinos. Una de las variables que influye en este consumo de energía es la presión hidráulica aplicada a la maza superior. Para determinar las posibilidades de reducir este consumo de energía se disminuyeron las presiones hidráulicas en un valor que no afectó la eficiencia del proceso de extracción. La investigación se realizó en dos tándems de seis molinos cañeros cada uno. Solo se variaron las presiones en los molinos de extracción en húmedo según un diseño estadístico de experimentos en bloques aleatorizados. Los resultados se analizaron mediante un análisis de varianza de clasificación doble. Las variables independientes fueron las presiones hidráulicas en los molinos intermedios; las dependientes fueron el $\%$ pol y \% de humedad del bagazo final. Se redujeron las presiones hidráulicas de los molinos intermedios 3.45 MPa en el Tándem 1 y 2.07 MPa en el Tándem 2. Se demostró que en las condiciones del experimento, el empleo de presiones hidráulicas de trabajo menores que las establecidas para cada tándem, no afectó significativamente el proceso de extracción de la sacarosa de la caña de azúcar, pero disminuyó $11.75 \%$ la demanda de energía del Tándem 1 y $8.17 \%$ para el Tándem 2.

\section{Descriptores:}

- presión hidráulica

- molino de caña de azúcar

- ahorro de energía

- tándem

- pol

- humedad 


\begin{abstract}
Among the areas with more energy consumption in a sugar factory is the tandem of mills. The applied hydraulic pressure on the superior mace is one of the variables that have influence on this energy consumption. Hydraulic pressures were decreased in a value that did not affect the extraction process efficiency to determine the possibilities of decreasing this energy consumption. The research was carried out in two sugar cane tandems of six mills. The pressures were only varied in the extraction mills in humid according to a statistical design of experiments in random blocks. The results were analyzed by means of the analysis of variance of double classification. The independent variables were the hydraulic pressures in the intermediate mills while the dependent variables were the $\%$ pol and \% humidity of the final bagasse. The hydraulic pressures of the intermediate mills were reduced $3.45 \mathrm{MPa}$ in the Tandem 1 and 2.07 MPa in the Tandem 2. It was demonstrated that under the conditions of the experiment, the employment of working hydraulic pressures smaller than the usually established ones for each tandem did not affect the extraction process of the sugar cane sucrose significantly, but decreased $11.75 \%$ the power demand on tandem 1 and $8.17 \%$ on tandem 2 .
\end{abstract}

\section{Introducción}

Unas de las vías para reducir los costos del proceso de producción de azúcar cruda de caña es disminuir las pérdidas de sacarosa en bagazo (Ortiz et al., 2008) y el consumo de energía en el tándem. Este consume alrededor de 30\% de la energía utilizada por la central azucarera (Gómez et al., 2006). Este consumo depende de muchos factores: eléctricos (Rosero y Ramírez, 2009; Gil et al., 2011), mecánicos (diseño del molino); operacionales (presiones hidráulicas, ajustes de los molinos, flujo y temperatura del agua de imbibición); las características de la caña que entra al molino (masa, fibra en caña, variedad y preparación), etcétera (Hugot, 1986).

El empleo de altas presiones hidráulicas en los molinos influye negativamente en la capacidad del molino, aumenta el costo operacional y de mantenimiento, así como la extracción de no azúcares (Hugot, 1986). Sin embargo, es una práctica común por los operarios del tándem aumentar las presiones hidráulicas aplicadas en los molinos y el nivel de imbibición para tratar de disminuir las pérdidas de azúcar en el bagazo residual, sin tener una base teórica o experimental para la selección de los valores a emplear (Gil et al., 2011).

El proceso de extracción de la sacarosa de la caña de azúcar en el tándem de molinos se puede considerar dividida en dos etapas: una de extracción en seco y otra en húmedo (Hamill, 1972). La de extracción en seco, propiamente dicha, puede estar constituida por una desmenuzadora y un primer molino, o solo por un primer molino o uno desmenuzador. Esta etapa es la que entrega el ali- mento (fibra + jugo) a la etapa de extracción en húmedo. La relación (masa de jugo/masa de fibra de caña), dependerá de la preparación de la caña y de la efectividad de la etapa de extracción en seco. Mientras menor sea el valor de esa relación en el tándem, mejores serán los resultados de la etapa de extracción en húmedo. Con la imbibición compuesta, el proceso de extracción en húmedo se acerca a uno de lixiviación a contracorriente (Hugot, 1986; Jenkins, 1971), donde cada etapa está constituida por el molino y el tramo de transportador que lo alimenta.

Los cálculos implicados en el diseño de procesos de lixiviación se conocen ampliamente (Treybal, 1977). Sin embargo, la lixiviación de la sacarosa en la etapa de extracción en húmedo en los molinos del tándem cañero, es un proceso muy complejo e ineficiente debido al gran poder de absorción del jugo por el bagazo y a la complejidad de la matriz porosa (Gil et al., 1999). En la operación del tándem prevalece el criterio mecánico para todos los molinos, aunque los procesos internos que ocurren en las etapas de extracción en seco y en húmedo son sustancialmente diferentes. Se ha caracterizado experimentalmente el proceso de compresión para diferentes superficies, demostrándose que la energía consumida en el proceso se ve afectada por el tipo de superficie de compresión (Díaz y Iglesias, 2012), pero no se refieren a valores de presiones hidráulicas. La primera etapa trata de extraer por compresión la mayor cantidad posible del jugo de las células abiertas retenido por el bagazo. En la segunda, se quiere lograr lixiviar mediante el proceso de imbibición la sacarosa del jugo retenido por el bagazo y llevar este último, en 
el último molino, a un porcentaje de humedad que permita utilizarlo económicamente como combustible.

$\mathrm{Al}$ no saber que se hayan realizado otras investigaciones que disminuyan las presiones hidráulicas en el tándem y que la tendencia es el aumento de estas; la investigación tuvo como objetivo demostrar que es posible disminuir apropiadamente las presiones hidráulicas en los molinos intermedios sin afectar la eficiencia del proceso de extracción y reducir el consumo de energía del tándem, proceso que han tratado de mejorar otros autores (Lloyd et al., 2010). Se partió de la hipótesis de que si el proceso en los molinos de extracción en húmedo del tándem cañero equivale a etapas de un proceso de lixiviación, entonces es posible disminuir las presiones hidráulicas a un valor que no afecte significativamente la eficiencia del proceso de extracción de la sacarosa, con la consiguiente disminución del consumo de energía del tándem cuando se opera a las nuevas presiones hidráulicas.

\section{Materiales y métodos}

La investigación se realizó en dos tándems de molinos cañeros de centrales diferentes con una capacidad de molida de 9200 t/día (800 000 @/día) para el Tándem 1 y 6900 t/día (600 000 @/día) para el Tándem 2. Ambos están compuestos por seis molinos de tres mazas, con cuarta maza o maza alimentadora y tolva alimentadora Donnelly en todos los molinos.

El trabajo experimental se desarrolló con base en un diseño de experimentos estadístico en bloques aleatorizados (Box et al., 1978). Se emplearon dos tratamientos: $P_{i}$ y $P_{i}-\Delta P$. Donde $P_{i}$ es la presión hidráulica de trabajo (PT) establecida por los directivos de cada central azucarera para aplicarse en cada uno de los i molinos de extracción en húmedo (i $=2$ a i $=5)$ del tándem cañero. $\mathrm{P}_{\mathrm{i}}-\Delta \mathrm{P}$ fue la presión hidráulica experimental (PE). Donde $\triangle \mathrm{P}$ tuvo un valor de $3,45 \mathrm{MPa}$ (500 PSI) para el Tándem 1 y de 2,07 MPa (300 PSI) para el Tándem 2. Estos valores de $\Delta \mathrm{P}$ fueron los autorizados por los directivos de cada central azucarera y no fueron mayores porque se piensa que el empleo de las altas presiones favorece el proceso de extracción de la sacarosa de la caña de azúcar (tabla 1).

Los intervalos de $2 \mathrm{~h}$ de trabajo del tándem se tomaron como bloques y, en cada intervalo, una hora para cada tratamiento, en cada central azucarera se realizaron los experimentos a las mismas horas, pero en distintos días. Considerando que la hora en que se realizan los experimentos y otras variables no controladas pudiera influir en los resultados, la aplicación de las presiones PT y PE se distribuyeron de forma aleatoria en los bloques. El diseño experimental quedó compuesto por nueve bloques, con dos tratamientos por bloque (tabla 2). Las variables controladas fueron el nivel de imbibición (masa de agua/masa de fibra en caña=2), temperatura del agua de imbibición $60^{\circ} \mathrm{C}$ y las presiones en el primero y último molino de cada tándem. Se consideraron como variables dependientes los \% pol y los $\%$ de humedad en el bagazo final.

\section{Metodología para el desarrollo de los experimentos}

1. Determinar el tiempo de retención de la caña en el tándem de molinos (es el intervalo de tiempo entre la entrada de la fibra de caña en el primer molino y la salida de bagazo en el último).

2. Ajustar las presiones hidráulicas de los molinos del tándem a las condiciones de operación según el diseño en bloques aleatorizados (tabla 1).

3. Tomar la muestra de bagazo a la salida del tándem. Para estabilizar las condiciones de molida, esperar un tiempo igual al doble del tiempo de retención de la caña en el tándem, antes de comenzar a tomar la muestra acumulativa de bagazo (Gil, 2011). El bagazo se muestreó a todo lo ancho del colchón empleando la toma de muestras. Se conformó una muestra

Tabla 1. Presiones a las que se desarrollaron los experimentos

\begin{tabular}{ccccc}
\hline \multirow{2}{*}{ Molinos } & \multicolumn{4}{c}{ Presión hidráulica, MPa } \\
\cline { 2 - 5 } & \multicolumn{2}{c}{ Tándem 1 } & \multicolumn{2}{c}{ Tándem 2 } \\
\cline { 2 - 5 } & PT & PE & PT & PE \\
\hline Molino 1 & 19.30 & 19.30 & 13.80 & 13.80 \\
Molino 2 & 17.90 & 14.45 & 16.50 & 14.43 \\
Molino 3 & 16.50 & 13.05 & 16.50 & 14.43 \\
Molino 4 & 16.50 & 13.05 & 16.90 & 14.83 \\
Molino 5 & 17.90 & 14.45 & 17.20 & 15.13 \\
Molino 6 & 19.30 & 19.30 & 17.20 & 17.20 \\
\hline
\end{tabular}

Tabla 2. Diseño estadístico de experimentos en bloques aleatorizados

\begin{tabular}{clcc}
\hline \multirow{2}{*}{ Núm. } & \multicolumn{1}{c}{ Bloques } & \multicolumn{2}{c}{ Tratamientos } \\
\cline { 2 - 4 } & \multicolumn{1}{c}{ Hora } & 1 & 2 \\
\hline 1 & $6: 00 \mathrm{am}-8: 00 \mathrm{am}$ & PE & PT \\
2 & $8: 00 \mathrm{am}-10: 00 \mathrm{am}$ & PE & PT \\
3 & $10: 00 \mathrm{am}-12: 00 \mathrm{~m}$ & PT & PE \\
4 & $12: 00 \mathrm{~m}-2: 00 \mathrm{pm}$ & PE & PT \\
5 & $2: 00 \mathrm{pm}-4: 00 \mathrm{pm}$ & PT & PE \\
6 & $4: 00 \mathrm{pm}-6: 00 \mathrm{pm}$ & PT & PE \\
7 & $10: 00 \mathrm{pm}-12: 00 \mathrm{am}$ & PE & PT \\
8 & $12: 00 \mathrm{am}-2: 00 \mathrm{am}$ & PT & PE \\
9 & $2: 00 \mathrm{am}-4: 00 \mathrm{am}$ & PT & PE \\
\hline
\end{tabular}


acumulativa de $5 \mathrm{~kg}$ de bagazo. Cada muestra de bagazo se guarda en una bolsa plástica herméticamente cerrada para evitar las pérdidas de humedad durante su traslado al laboratorio.

4. En el laboratorio la muestra de bagazo se homogeneizó y se redujo de tamaño por el método de paladas alternas (Gil, 2011), para conformar dos muestras para las determinaciones de pol y de humedad.

5. Determinar pol y humedad a las muestras de bagazo, según el procedimiento establecido en el laboratorio azucarero.

6. Realizar el mismo procedimiento para todos los experimentos (sea a las PT o las PE).

Los resultados experimentales se examinaron mediante un Análisis de Varianza de Clasificación Doble (Box et al., 1978) para analizar el efecto de los tratamientos y de la posible influencia de los bloques en los resultados para un nivel de confianza de $95 \%$.

\section{Metodología para el cálculo del ahorro de energía en el tándem}

Para el cálculo de la demanda de potencia de los molinos del tándem se empleó la ecuación de Hugot (1986). Es una de las más completas por incluir en las variables al coeficiente de reabsorción.

$N=\frac{n D}{\eta}\left\{P\left[0.4 \frac{6 r-5}{\sqrt{r}(1+\sqrt{r-1})} \sqrt{\varepsilon_{A}}+0.075\right]+4 L\right\}$

donde:

$N=$ potencia demandada por el molino $(\mathrm{kW})$

$n=$ velocidad de rotación de la masa superior (rpm)

$D=$ diámetro medio de los cilindros (m)

$\eta=$ rendimiento de la transmisión

$\mathrm{P}=$ fuerza hidráulica total aplicada $\left(\mathrm{t}_{\text {cortas }}\right)$

$r=$ factor de reabsorción

$\varepsilon_{A}=$ abertura específica de salida

$L=$ longitud de las mazas $(\mathrm{m})$

En los dos tándems en estudio se utilizan motores independientes para el movimiento de los transportadores intermedios. Esta medida hace más flexible la operación de los transportadores. Para adaptar la ecuación (1) fue necesario restarle a la demanda total de potencia, el valor de la demanda de potencia del movimiento de los transportadores intermedios. Aunque esa potencia se sigue demandando, ya no se puede tener en cuenta como carga del motor principal.
La cuarta maza o alimentador forzado recibe el movimiento desde la maza superior del molino mediante una corona habilitada al efecto. Si a la ecuación planteada por Hugot en (1) se le resta la potencia demandada por el movimiento de los transportadores intermedios y se le añade la potencia demandada por la cuarta maza, la ecuación resultante es la siguiente

$N=\frac{n D}{\eta}\left\{P\left[0.4 \frac{6 r-5}{\sqrt{r}(1+\sqrt{r-1})} \sqrt{\varepsilon_{A}}+0.076\right]+2 L\right\}$

Para el caso de los primeros molinos Hugot recomienda que el primer coeficiente dentro del corchete en la expresión (2) sea 0.3 en lugar de 0.4 , debido al trabajo de preparación adicional que debe realizar este equipo (Hugot, 1986). Esta recomendación es para el caso en que el primer molino esté precedido por cuchillas, como ocurre en el caso que se analiza. Por último, la ecuación para trabajar en todos los molinos será la ecuación (3).

$N=\frac{n D}{\eta}\left\{P\left[0.3 \frac{6 r-5}{\sqrt{r}(1+\sqrt{r-1})} \sqrt{\varepsilon_{A}}+0.076\right]+2 L\right\}$

La disminución en la demanda de potencia por molino implica un ahorro de energía. Para el caso específico que se analiza, se utilizó un promedio de 100 días de zafra para calcular el ahorro de energía según la ecuación (4)

$A e=\left(\sum_{i=1}^{6} N_{t r a, i}-\sum_{i=1}^{6} N_{\mathrm{e} x p, i}\right) * 100 \frac{\text { día }}{z a f r a} * 24 \frac{h}{d i ́ a}$

donde:

Ae $=$ ahorro de energía de cada tándem

$\sum_{i=1}^{6} N_{t r a, i}=\begin{aligned} & \text { potencia demandada por el tándem a las } \\ & \text { presiones de trabajo }\end{aligned}$ $\sum_{i=1}^{6} N_{\text {exp }, i}=\begin{aligned} & \text { potencia demandada por el tándem a las } \\ & \text { presiones experimentales. }\end{aligned}$

\section{Resultados y discusión}

En el periodo de los experimentos, el molino 1 del Tándem 2 se operó con una presión hidráulica de 13,80 MPa debido a algunas fallas mecánicas que presentó. Esto afectó la eficiencia del tándem durante ese periodo, pero no fue de interés para la investigación porque no influyó en los resultados de los experimentos (tabla 3). 
Los resultados fueron sometidos a una prueba de bondad de ajuste para una distribución normal y se comprobó que la hipótesis de que los datos proceden de distribuciones normales no se rechaza al nivel de significancia de 0.05 , ya que el sesgo estandarizado y la curtosis estandarizada están en el rango de -2 a +2 (tabla 4).

Para determinar si la disminución de la presión hidráulica influyó sobre el \% de pol y \% de humedad del bagazo residual y si estos resultados tuvieron influencia también de los bloques, se realizó un análisis de varianza (ANOVA) de clasificación doble a un nivel de significancia $\alpha=0.05$. Los resultados del ANOVA respecto a la probabilidad de aceptar las hipótesis de igualdad de las medias y de la no influencia de los bloques aparecen en la tabla 5.
Del ANOVA, si las probabilidades calculadas son mayores que 0.05 , se infiere que no existieron diferencias significativas entre los bloques, por lo que las características que estos tuvieron durante los experimentos no influyeron significativamente en los resultados en los parámetros del bagazo final cuando se trabajó con las presiones hidráulicas tradicionales de trabajo y las experimentales en los molinos de extracción en húmedo de cada tándem.

Estos resultados indican que el proceso de extracción en los molinos de extracción en húmedo debe estudiarse como un proceso de lixiviación-compresión y que la lixiviación influye fuertemente en los resultados del tándem. Basado en el proceso de lixiviación es posible realizar mejoras en el tándem, que permitan trabajar aún a menores presiones con igual o mayor eficiencia en el proceso de extracción.

\begin{tabular}{ccccccccc}
\hline & \multicolumn{9}{c}{ Tándem 1 } & \multicolumn{5}{c}{ Tándem 2 } \\
\cline { 2 - 8 } Núm. Exp & \multicolumn{3}{c}{ Pol (\%) } & \multicolumn{2}{c}{ Humedad $(\%)$} & \multicolumn{2}{c}{ Pol (\%) } & \multicolumn{2}{c}{ Humedad (\%) } \\
\cline { 2 - 8 } & PT & PE & PT & PE & PT & PE & PT & PE \\
\hline 1 & 2.48 & 2.50 & 48.60 & 48.20 & 2.91 & 2.78 & 50.04 & 52.60 \\
2 & 2.17 & 2.25 & 50.60 & 49.20 & 2.70 & 2.70 & 50.85 & 51.84 \\
3 & 2.20 & 2.79 & 49.60 & 48.60 & 2.66 & 2.70 & 50.90 & 51.64 \\
4 & 2.56 & 2.34 & 50.60 & 49.80 & 2.75 & 2.76 & 52.89 & 52.32 \\
5 & 2.26 & 2.45 & 50.10 & 49.50 & 2.84 & 2.79 & 52.89 & 52.00 \\
6 & 2.32 & 2.17 & 48.90 & 49.10 & 2.75 & 2.74 & 51.30 & 50.22 \\
7 & 2.16 & 2.33 & 49.40 & 50.10 & 2.83 & 2.83 & 52.42 & 50.92 \\
8 & 2.21 & 2.25 & 48.80 & 48.90 & 2.87 & 2.76 & 51.72 & 50.87 \\
9 & 2.23 & 2.12 & 49.20 & 49.50 & 2.74 & 2.80 & 52.04 & 51.52 \\
\hline
\end{tabular}

Tabla 3. Valores de los parámetros de calidad del bagazo en el experimento

\begin{tabular}{ccc}
\hline Tándem 1 & Sesgo Estandarizado & Curtosis Estandarizada \\
\hline Pol, PT & 1.54461 & 0.239273 \\
Pol, PE & 1.49314 & 1.08108 \\
Humedad, PT & 0.510449 & -0.806057 \\
Humedad, PE & -0.320386 & -0.201301 \\
Tándem 2 & Sesgo Estandarizado & Curtosis Estandarizada \\
Pol, PT & 0.122487 & -0.682334 \\
Pol, PE & -0.235163 & -0.392691 \\
Humedad, PT & -0.276563 & -0.579853 \\
Humedad, PE & -0.518596 & -0.287648 \\
\hline
\end{tabular}

Tabla 4. Prueba de normalidad de los valores experimentales de los \% pol y \% humedad del bagazo 


\begin{tabular}{ccc}
\hline & $\begin{array}{c}\text { \%PolPT vs \%PolPE } \\
\text { Probabilidad }\end{array}$ & $\begin{array}{c}\text { \%Humedad PT vs \% Humedad PE } \\
\text { Probabilidad }\end{array}$ \\
\hline Tándem 1 & & \\
Tratamiento & 0.4226 & 0.1992 \\
Bloque & 0.4366 & 0.0798 \\
Tándem 2 & & \\
Tratamiento & 0.3522 & 0.7809 \\
Bloque & 0.0560 & 0.5976 \\
\hline
\end{tabular}

Tabla 5. Resultados del Análisis de Varianza

\begin{tabular}{ccccc}
\hline \multirow{2}{*}{ Molinos } & \multicolumn{4}{c}{ Demanda de potencia, kW } \\
& De trabajo & Experimentales & De trabajo & Experimentales \\
\hline Molino 1 & 564.27 & 564.27 & 341,76 & 341,76 \\
Molino 2 & 467.84 & 382.24 & 559,99 & 494,43 \\
Molino 3 & 435.41 & 349.72 & 538,44 & 475,54 \\
Molino 4 & 425.86 & 342.16 & 619,42 & 545,61 \\
Molino 5 & 458.83 & 375.00 & 590,57 & 525,44 \\
Molino 6 & 487.10 & 487.10 & 622,89 & 622,89 \\
Total & 2833.31 & 2500.41 & 3273,07 & 3005,68 \\
\hline Diferencia & \multicolumn{3}{c}{267.39} \\
\hline
\end{tabular}

Tabla 6. Potencia demandada por molinos

Considerando que el agua de imbibición o los jugos que se vierten sobre el colchón de bagazo, son absorbidos completamente por la capa superficial (Gil et al., 1999), es posible introducir mejoras tecnológicas que contribuyan a un mejor mezclado del líquido de imbibición con el jugo rico en sacarosa que retiene la matriz porosa del bagazo. Esto contribuiría a aumentar la eficiencia de la lixiviación en el molino y permitiría operarlo con menores presiones hidráulicas, porque el jugo retenido por la fibra sería jugo diluido y no el jugo sin diluir debido al insuficiente mezclado. Además se conoce que la presión efectiva de los molinos intermedios es mucho menor que las presiones empleadas tradicionalmente (Gil, 2011). El empleo de menores presiones causaría una disminución en el índice de roturas, menos desgaste de las partes móviles y menor consumo energético (Hugot, 1986).

\section{Ahorro de energía debido a la disminución en la demanda de potencia}

Por medio de la ecuación (3) se calculó la potencia demandada por el motor eléctrico de cada molino. En el Tándem 1 hubo una disminución en la demanda de potencia de $332.90 \mathrm{~kW}$ al disminuir las presiones de trabajo hasta las experimentales. En el Tándem 2 la dis- minución en la demanda de potencia fue de $267.39 \mathrm{~kW}$ (tabla 6).

El ahorro de energía eléctrica se calculó con la ecuación (4) con base en 100 días de zafra. Para el Tándem 1 el ahorro de energía fue 798.96 MWh y para el Tándem 2, 631.74 MWh, para un ahorro total de energía de 1.44 GWh.

\section{Conclusiones}

1. Es posible disminuir las presiones hidráulicas aplicadas en los molinos de extracción en húmedo de un tándem cañero, sin afectar el \% pol y \% de humedad en el bagazo final establecidos por la central azucarera como parámetros de eficiencia del proceso de extracción en una magnitud que debe ser evaluada para cada tándem.

2. La posibilidad de disminuir las presiones hidráulicas de trabajo en los molinos de extracción en húmedo de un tándem cañero sin afectar la eficiencia del proceso de extracción, indica que en ellos hay que considerar el proceso combinado lixiviación-compresión y no solo de extracción del jugo por compresión, dando la posibilidad de introducir mejoras tecnológicas que contribuyan a mejorar el proceso de lixiviación. 
3. Es posible lograr considerables ahorros de energía en el tándem de molinos operando los molinos de extracción en húmedo a menores presiones hidráulicas que las empleadas tradicionalmente sin afectar los parámetros de calidad del bagazo final.

\section{Referencias}

Box E.P., Hunter W.G., Hunter J.S. Statistics for Experimenters. An Introduction to Design, Data Analysis and Model Building, editorial John Wiley \& Sons, 1978.

Díaz A., Iglesias C. Dinámica del proceso de extracción de jugo a compresión de la caña de azúcar para la producción de panela. Revista Ciencias Técnicas Agropecuarias, volumen 21 (número 2), abril-junio 2012 [en línea] [fecha de consulta: 27 de febrero de 2013]. Disponible en: http://scielo.sld.cu/pdf/rcta/ v21n2/rcta14212.pdf. ISSN 2071-0054.

Gil J.A. y Gil J.M. Capacidad de retención del jugo por el bagazo. Revista Tecnología Química, volumen 19 (número 1), eneroabril 1999 [en línea] [fecha de consulta: 27 de febrero de 2013]. Disponible en: http://ojs.uo.edu.cu/index.php/tq/article/ download/1731/1271. ISSN 2224-6185.

Gil J.M. Intensificación del proceso de extracción de la sacarosa de la caña de azúcar con el uso de surfactantes aniónicos en el agua de imbibición, (tesis de doctorado en ciencia técnicas), Cuba, Universidad de Oriente, 2011. [en línea] [fecha de consulta: 26 de septiembre de 2012]. Disponible en: http://www.eumed.net/ tesis/2011/jmgo/index.htm

Gil J.M., Nápoles O., Remedios P. et al. Evaluación del consumo de potencia del motor eléctrico del sexto molino de un tándem cañero. Revista Tlatemoani, (número 5), marzo 2011 [en línea] [fecha de consulta: 2 de octubre de 2012]. Disponible en: http://www.eumed.net/rev/tlatemoani/05/ogcsp.pdf. ISSN 1989-9300.

Gómez J., De Armas M., Gil A. Competitividad y eficiencia en las oportunidades de ahorro de energía eléctrica en la industria azúcar. Revista Centro Azúcar, volumen 33 (número 1), eneromarzo 2006 [en línea] [fecha de consulta: 2 de octubre de 2012]. Disponible en: http://centroazucar.qf.uclv.edu.cu/media/articulos/PDF/2006/1/13.pdf. ISSN 2223-4861.

Hamill T.M. Wet Milling Extraction Process and Apparatus Therefor. Patente 36695931 United States Patent Office, 1972.
Hugot E. Handbook of Cane Sugar Engineering, editorial Elsevier, Amsterdam, 1986.

Jenkins G. Introducción a la tecnología del azúcar de caña, editorial Ciencia y Técnica, La Habana, 1971.

Lloyd T., Eastment S., Mitchell P. Milling Train Maceration Control Utilising Nir Technology. Australian Society of Sugar Cane Technologists, volumen 32, 2010 [en línea] [fecha de consulta: 27 de febrero de 2013]. Disponible en: http://www.assct.com. au/media/pdfs/M\%2016\%20Lloyd.pdf.

Ortiz F., Tobón L.G., Alvarado A.M., et al. Disminución de pérdidas de sacarosa en la elaboración de meladura en un ingenio azucarero. Revista de la Ingeniería Industrial, volumen 2 (número 1), 2008 [en línea] [fecha de consulta: 27 de febrero de 2013]. Disponible en: http://academiajournals.com/downloads/OrtizTobon.pdf. ISSN 1940-2163.

Rosero E., Ramirez J. Modelado y control de molinos de caña de azúcar usando accionamientos eléctricos. Rev. Iberoamericana de Automática e Informática Industrial, volumen 6 (número 3), julio 2009. [en línea] [fecha de consulta: 2 de octubre de 2012]. Disponible en: http://zl.elsevier.es/es/revista/revista-iberoamericana-automatica-e-informatica-331/pdf/90083496/ S300/. ISSN 1697-7912.

Treybal R. Operaciones con transferencia de masa, 2ª ed., McGrawHill, Madrid, 1977,

\section{Este artículo se cita: \\ Citación estilo Chicago}

Corrales-Suárez, Jorge Michel, José Marcos Gil-Ortiz, Pedro Dionisio Remédios-Castaneiras, Yurisleydis Masjuan-Leyva, José Alexander Gil-Ceballo. Operación de los molinos del tandem cañero a dos niveles de presión hidráulica. Ingeniería Investigación y Tecnología, XVI, 01 (2015): 123-130.

\section{Citación estilo ISO 690}

Corrales-Suárez J.M., Gil-Ortiz J.M., Remédios-Castaneiras P.D., Masjuan-Leyva Y., Gil-Ceballo J.A. Operación de los molinos del tandem cañero a dos niveles de presión hidráulica. Ingeniería Investigación y Tecnología, volumen XVI (número 1), enero-marzo 2015: 123-130p. 


\section{Semblanza de los autores}

Jorge Michel Corrales-Suárez. Ingeniero mecánico graduado en la Universidad de Camagüey, Cuba. Actualmente trabaja como profesor e investigador en el Centro de Estudio de Energía y Procesos Tecnológicos de la Universidad de Las Tunas, Cuba. Profesor de termodinámica y mecánica de los fluidos-máquinas de flujo. Sus líneas de investigación son la eficiencia energética y los procesos tecnológicos, candidato a doctor en ciencias técnicas.

José Marcos Gil-Ortiz. Licenciado en física, ingeniero químico, master en ingeniería química y doctor en ciencias técnicas. Actualmente trabaja como profesor e investigador en el Centro de Estudio de Energía y Procesos Tecnológicos de la Universidad de Las Tunas, Cuba. Profesor de operaciones unitarias de los procesos químicos. Sus líneas de investigación son: El proceso de extracción en tándem de molinos cañeros y eficiencia energética de procesos.

Pedro Dionisio Remédios-Castañeiras. Ingeniero mecánico, master en ingeniería química y doctor en ciencias técnicas. Actualmente trabaja como profesor e investigador en el Centro de Estudio de Energía y Procesos Tecnológicos de la Universidad de Las Tunas, Cuba. Profesor de operaciones unitarias de los procesos químicos. Sus líneas de investigación son: mecánica de los fluidos y sistemas de bombeo, flujos viscosos en la industria azucarera y eficiencia energética de procesos.

Yurisleydis Masjuan-Leyva. Ingeniera industrial graduada en la Universidad de Las Tunas. Cuba. Actualmente se desempeña como especialista energética en el Departamento de Ingeniería y Desarrollo de la Empresa Logística Agropecuaria Las Tunas.

José Alexander Gil-Ceballo. Ingeniero químico, máster en informática aplicada. Colaborador del Centro de Estudio de Energía y Procesos Tecnológicos de la Universidad de Las Tunas, Cuba. Línea de investigación: gestores de negocios. 